\title{
CXCL5 expression in tumor tissues is associated with poor prognosis in patients with pancreatic cancer
}

\author{
BIN WU, JING WANG, XIAOGUANG WANG, MINGYUAN ZHU, FEI CHEN, \\ YIYU SHEN and ZHENGXIANG ZHONG
}

\begin{abstract}
Department of Hepatobiliary Surgery, The Second Affiliated Hospital of Jiaxing University, Jiaxing, Zhejiang 314000, P.R. China
\end{abstract}

Received April 10, 2020; Accepted August 25, 2020

DOI: $10.3892 / \mathrm{ol} .2020 .12120$

\begin{abstract}
Immunotherapy based on the tumor microenvironment is a feasible method for treating cancer; therefore, it is necessary to investigate the immune microenvironment of pancreatic cancer and the influencing factors of the immune microenvironment. Chemokines are an important factor affecting the tumor immune microenvironment. In the present study, chemokines or chemokine receptors were screened to identify those differentially expressed in pancreatic cancer compared with normal controls and associated with patient prognosis. Chemokines or chemokine receptors that are differentially expressed in pancreatic cancer tumor tissues were initially screened using the Gene Expression Omnibus database. Next, survival analysis was performed using GEPIA, a website based on The Cancer Genome Atlas (TCGA) database. Immunohistochemical staining of CXCL5 was performed in tissue microarrays (TMAs) containing 119 cases of pancreatic cancer. Histochemistry score (H-SCORE) was used to evaluate the expression of CXCL5. Next, association analysis of the H-SCORE of CXCL5 and the clinical characteristics of patients was performed, as well as Kaplan-Meier survival and Cox multivariate regression analyses. The results of the bioinformatics analysis demonstrated that CXCL5 was highly expressed in pancreatic cancer tissues. High expression of CXCL5 in pancreatic cancer tissues was associated with a poor prognosis in patients in TCGA cohort. The expression level of CXCL5 in tumor tissues was significantly higher compared with that in adjacent peritumoral normal tissues in the immunohistochemical analysis. There was no significant association between CXCL5 expression in pancreatic cancer
\end{abstract}

Correspondence to: Professor Zhengxiang Zhong or Professor Yiyu Shen, Department of Hepatobiliary Surgery, The Second Affiliated Hospital of Jiaxing University, 1518 Huancheng North Road, Jiaxing, Zhejiang 314000, P.R. China

E-mail: 13505739209@139.com

E-mail: syjdr@163.com

Key words: CXCL5, tumor microenvironment, prognosis, pancreatic cancer tumor tissues and clinicopathological factors. Patients with pancreatic cancer with high CXCL5 expression had a poor prognosis, as determined by Kaplan-Meier survival analysis based on the TMA dataset. The results of Cox multivariate regression analysis showed that CXCL5 was an independent factor for a poor prognosis in patients with pancreatic cancer. In conclusion, the results of the present study revealed that the chemokine CXCL5 was highly expressed in pancreatic cancer tissues; high CXCL5 expression was associated with a poor prognosis in patients with pancreatic cancer.

\section{Introduction}

Pancreatic cancer, particularly pancreatic ductal adenocarcinoma (PDAC) is extremely malignant, with a 5-year overall survival rate of only $\sim 9 \%$ (1). The symptoms of pancreatic cancer are not clear in the early stages, and most patients present with local invasion or distant metastasis at the time of diagnosis. Therefore, the surgical resection rate is low, with a previous study reporting a resection rate of $10-20 \%$ (2). Patients with pancreatic cancer often experience metastasis and recurrence following surgery, and the prognosis is poor (3). Clinical data have demonstrated that pancreatic cancer remains incurable and that traditional chemoradiotherapy cannot prolong the survival of patients (3-5). In the past, tumor cells were the main subject of tumor research, particularly in studies investigating the role of genes and signaling pathways in tumorigenesis and tumor progression; these studies were used to find potential therapeutic targets for pancreatic cancer, but no major targets have been identified (6). Recent studies have reported that the tumor microenvironment serves an important role in tumorigenesis and development $(7,8)$. These studies have suggested that the malignant biological behavior of tumors may be regulated by the tumor microenvironment (9-11). Immunotherapy based on the tumor microenvironment is a feasible treatment method; therefore, it is necessary to investigate the immune microenvironment of pancreatic cancer and its influencing factors.

The components of the tumor microenvironment include tumor cells, interstitial cells, immune cells and cell regulatory factors secreted by cells in the microenvironment or secreted by other cells and transported to the microenvironment; the most important of these components may be tumor immune 
cells, including B lymphocytes, T lymphocytes, neutrophils, macrophages, dendritic cells, and other immune cells that infiltrate tumor tissues $(7,8)$. Previous studies have shown that the tumor microenvironment of pancreatic cancer is extremely complex $(12,13)$. Tang et al $(14)$ have reported that the level of $\mathrm{CD} 8^{+} \mathrm{T}$ lymphocyte infiltration in pancreatic tumor tissues is lower compared with that in adjacent normal tissues. Treg cells, which highly infiltrate tumor tissues, inhibit the antitumor immunity of $\mathrm{CD}^{+} \mathrm{T}$ lymphocytes, which may be associated with tumor immune escape (14). In another study, Liu et al (15) reported that in 92 patients with pancreatic cancer, the number of infiltrating $\mathrm{CD}^{+}$and $\mathrm{CD} 8^{+} \mathrm{T}$ lymphocytes in the adjacent normal tissues of pancreatic cancer was higher compared with that in the tumor tissues, but $\mathrm{CD}^{+}{ }^{+} \mathrm{FOXP}^{+}$Treg cells were more abundant in the tumor tissues. The survival analysis showed that high infiltration of $\mathrm{CD} 8^{+} \mathrm{T}$ lymphocytes in adjacent normal tissues and low infiltration of $\mathrm{CD}^{+} \mathrm{T}$ cells in tumor tissues were good independent prognostic factors (15). Therefore, we hypothesized that immune cell infiltration is an important part of the tumor microenvironment.

Tumors are considered to be 'organs' composed of different types of cells (7). Compared with normal tissues, the information interactions between cells in the tumor microenvironment are more complicated (8). A previous study has demonstrated that these interactions are associated with the occurrence and progression of tumors (16). Chemokines are important mediators of interactions between different types of cells in the tumor microenvironment (17). Chemokines not only act as chemotactic molecules for targeted migration, but also serve an important regulatory role in various physiological and pathological processes of the cell (including infection, wound repair, inflammatory response, tumorigenesis and invasion) (18). Chemokines in the tumor microenvironment may act on tumor cells and other stromal cell components, including neutrophils, macrophages, lymphocytes and fibroblasts, to promote or inhibit tumor progression $(19,20)$. Furthermore, there may also be negative chemokines that support a tumor microenvironment with no T lymphocyte infiltration (21). A lack of T lymphocyte infiltration in the microenvironment may lead to a poor prognosis (22).

In the present study, chemokines or chemokine receptors were screened to identify those differentially expressed in pancreatic cancer compared with normal controls and associated with patient prognosis.

\section{Materials and methods}

Public datasets from the gene expression omnibus (GEO) database. The gene expression microarray and corresponding clinical data of patients with pancreatic cancer were obtained from the GEO database (https://www.ncbi.nlm.nih. gov/geo/). The dataset numbers were GSE56560 $(23,24)$ and $\operatorname{GSE} 28735(25,26)$.

Screening of differentially expressed (DE) chemokines and chemokine receptors. Initially, analysis of DE genes between pancreatic tumor tissues and normal tissues was performed using the 'limma' package (http://www.bioconductor. org/packages/release/bioc/html/limma.html) in R $(27,28)$, according to the screening criteria (adjusted $\mathrm{P} \leq 0.10$ and $\operatorname{llog}$ fold-changel $\geq 1.5$ ). DE chemokines and $\mathrm{DE}$ chemokine receptors were determined by overlapping the DE genes with a dataset containing all chemokines and their receptors; this analysis was performed using the 'VennDiagram' package in $R(29)$.

Survival analysis of the TCGA dataset. The survival analysis was performed on the Gene Expression Profiling Interactive Analysis website (GEPIA; http://gepia.cancer-pku.cn/). The data for 178 patients with pancreatic cancer analyzed on the GEPIA website were obtained from The Cancer Genome Atlas (TCGA, https://cancergenome.nih.gov/).

Patients and tissue microarray. Two commercial tissue microarray (TMA) chips consisting of 290 points were used. Of these, there were 128 pairs of intra-tumoral tissues and adjacent peri-tumoral normal tissues (Shanghai Outdo Biotech, Shanghai, China). All patients from the TMA underwent surgical treatment. If the tumor was located in the head of the pancreas, pancreaticoduodenectomy was performed. If the tumor was located in the tail of the pancreas, pancreatic body tail resection was performed. All patients had a surgical pathological diagnosis. Clinicopathological data included pathological type, operation time, survival status, follow-up time, survival time, tissue code, tumor organ, sex, age, patient's main complaint, tumor history, family tumor history, distant metastasis site, whether it was a primary organ or metastatic, pathological classification, pathological grade, tumor size, tumor site, description of the tumor, texture of the tumor, tumor capsular invasion, pathological morphology, tumor boundary, vascular invasion, lymph node invasion, number of total and invaded lymph nodes, $\mathrm{T}$ stage, $\mathrm{N}$ stage, $\mathrm{M}$ stage and American Joint Committee on Cancer stage (30). All patients received conventional chemotherapy following surgery and were followed up regularly. The follow-up information came from outpatient follow-up review or telephone follow-up. Overall survival (OS) time refers to the time between surgery and death, with OS as a prognostic indicator of survival.

Immunohistochemical staining and interpretation. Immunohistochemical staining was performed on tissue sections deparaffinized in xylene with an UltraSensitive SP Mouse/Rabbit IHC kit (cat. no. KIT-9720; Maxim Biotech, Inc.) according to the manufacturer's instructions and an IHC Biotin Block kit (cat. no. BLK-0002; Maxim Biotech, Inc.) by an automated immunostainer (cat. no. ST5010; Leica Microsystems, Inc.). The protocol for the IHC Biotin Block kit is as follows: After the antigen retrieval, the PBS was removed, Avidin solution (reagent A, $50 \mu \mathrm{l}$ ) was added dropwise to the TMA and incubated at room temperature for $10 \mathrm{~min}$. Subsequently, the TMA was rinsed twice with PBS (3 min per rinse); the PBS was removed, and d-Biotin Solution (reagent B, $50 \mu \mathrm{l}$ ) was added dropwise to the TMA and incubate for $10 \mathrm{~min}$ at room temperature, followed by further two rinses with PBS (3 min per rinse). An anti-human CXCL5 monoclonal antibody (1:50; cat. no. MAB 254-100; R\&D Systems) was used for analysis. A fully automatic digital pathology slice scanner (Aperio; Leica Microsystems, Inc.) was used to scan the immunohistochemically stained TMA chips to obtain a digital image. The microscopic images were 
imported as digital photo files analyzed using QuantCenter in Pannoramic Viewer software 1.15.4 (3DHISTECH, Ltd.). Following preliminary observation, the unsatisfactory points of TMA in the sectioning and staining images were eliminated. TMA images were analyzed by two independent pathologists who were blinded to the patient's clinical data. Histochemistry score (H-SCORE) was used to evaluate the expression of CXCL5. H-SCORE is a scoring method for tissue immunohistochemical results that reflects the positive ratio and the positive intensity. The following formula was used: H-SCORE $=\sum$ (PI x I) $=($ percentage of cells of weak intensity $\mathrm{x} 1)+($ percentage of cells of moderate intensity $\mathrm{x} 2)$ + (percentage of cells of strong intensity $\times 3$ ). In the formula, PI represents the percentage of positive cells to the total number of cells in this position and I represents the intensity of staining. The H-SCORE is 0-300, with a higher score representing stronger positive staining. The H-SCORE for each patient was calculated, and all patients were divided into two groups (low and high CXCL5) according to the cut-off value. The H-SCORE cut-off value was determined using X-tile software version 3.6.1 (Yale University), which determines the cut-off value by calculating the P-value corresponding to each possible cut-off value; the cut-off corresponding to the lowest $\mathrm{P}$-value is considered the best. The calculations revealed that the best cut-off value for the H-SCORE was 76.83 .

Statistical analysis. In the present study, Student's t-test was used for comparing quantitative data, and the $\chi^{2}$ test, Fisher's exact test or likelihood ratio test were used for qualitative data. The Kaplan-Meier method was used to generate survival curves, and the log-rank method was used to compare the differences in survival rates between the groups. Multivariate analysis was performed using the Cox multivariate regression analysis model. The clinicopathological factors with significant associations $(\mathrm{P}<0.1)$ in the aforementioned Kaplan-Meier survival analysis were subjected to Cox multivariate analysis. The statistical and mapping software used in the present study were SPSS 22.0 (IBM Corp.), GraphPad Prism 6.02 (GraphPad Software, Inc.), Adobe Photoshop CS5, and Adobe Illustrator CS6 (both from Adobe Systems, Inc.). $\mathrm{P}<0.05$ was considered to indicate a statistically significant difference.

\section{Results}

CXCL5 is differentially expressed in two datasets from the GEO database. A total of $890 \mathrm{DE}$ genes were identified between the pancreatic tumor tissues and the normal tissues in the GSE56560 dataset, and 116 DE genes were identified in the GSE28735 dataset. A total of 10 DE chemokines or DE chemokine receptors were identified in the GSE56560 dataset, and only one (CXCL5) was identified in the GSE28735 dataset. Therefore, CXCL5 was selected for further analysis (Fig. 1).

Patients with pancreatic cancer with high CXCL5 expression in TCGA dataset have a poor prognosis. A total of 198 patients from TCGA dataset were included in the present study; they were divided into groups of 89 patients with low CXCL5 expression and 89 patients with high CXCL5 expression according to the median value of CXCL5 expression.

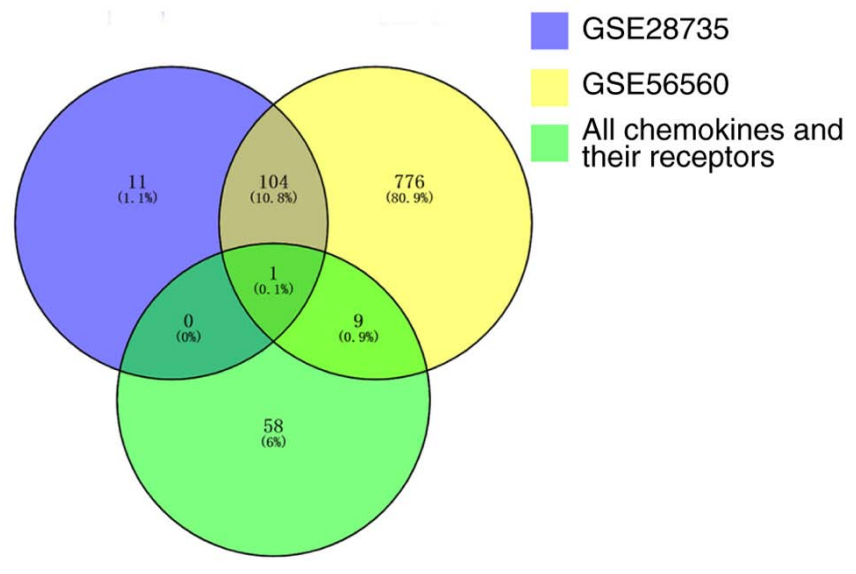

Figure 1. Venn diagram of DE chemokines or DE chemokine receptors. CXCL5 was identified in all datasets and was selected for further analysis. DE, differentially expressed.

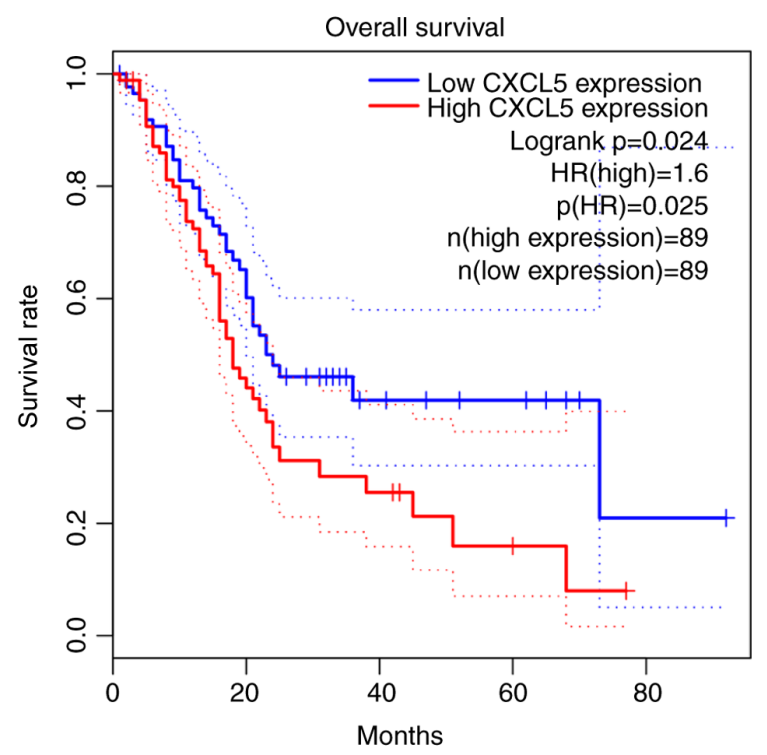

Figure 2. Kaplan-Meier survival analysis of patients based on The Cancer Genome Atlas data. HR, hazard ratio.

The prognosis of the patients in the low CXCL5 group was significantly improved compared with that of the patients in the high CXCL5 group ( $\mathrm{P}=0.024$; Fig. 2).

Characteristics of 119 patients from TMA dataset. Following removal of unsatisfactory points of TMA chips in the sectioning and staining images, a total of 119 patients with pancreatic cancer were included in the study. Samples of tumor and corresponding peritumoral healthy tissue were collected from each patient. Table I presents the clinicopathological characteristics of the patients with pancreatic cancer.

Expression of CXCL5 in the TMA dataset. The mean $\mathrm{H}-\mathrm{SCORE}$ of CXCL5 expression in pancreatic cancer tissues in the TMA was 94.58, and the 95\% confidence interval (CI) was 89.35-99.82. The H-SCORE in adjacent peri-tumoral normal tissues was 57.14, and the 95\% CI was 54.58-59.69. 
Table I. Patient demographics and clinicopathological factors.

\begin{tabular}{ll}
\hline Factors $\quad$ No. of patients
\end{tabular}

\section{Age, years}

$<70$

$\geq 70$

Sex

Male

Female

Grade

II

III

IV

IV

Tumor size, $\mathrm{cm}$

$\leq 4$

$>4$

Tumor site

Head

Other

Vascular invasion

No

Yes

$\mathrm{T}$

$\mathrm{T} 1$

$\mathrm{T} 2$

T3

T4

$\mathrm{N}$

N0

N1

N2

$\mathrm{M}$

M0

87

M1

TNM stage ${ }^{\mathrm{a}}$

I

II

63

III

11

IV

aTNM stage of patients with pancreatic adenocarcinoma according to the American Joint Commission on Cancer guidelines (8th edition) (30). T, tumor; N, node; M, metastasis; TNM, Tumor-Node-Metastasis.

The expression level of CXCL5 in tumor tissues was significantly higher than that in adjacent peritumoral healthy tissues, and the difference was statistically significant $(\mathrm{P}<0.0001$; Fig. 3A). Typical staining is presented in Fig. 3B (pancreatic cancer tissues) and $\mathrm{C}$ (adjacent normal tissues).
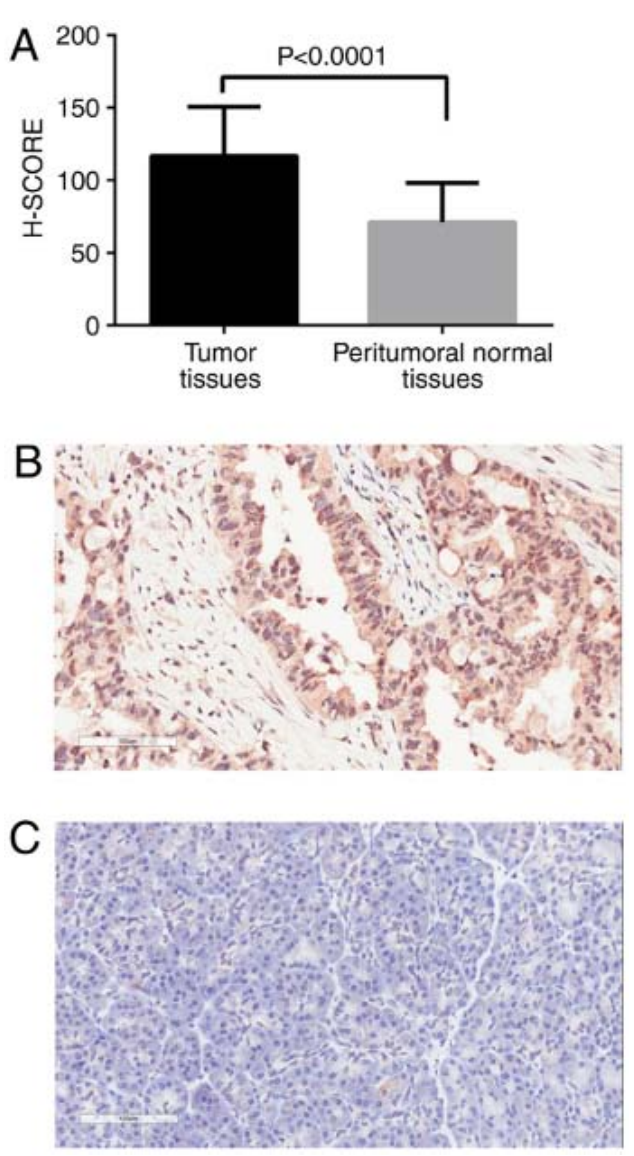

Figure 3. Expression of CXCL5 in pancreatic cancer. (A) Statistical comparison of CXCL5 expression levels (H-SCORE) in pancreatic cancer tumor tissues and adjacent peritumoral normal tissues. (B) CXCL5 was highly expressed in pancreatic cancer tumor tissues. (C) Low expression of CXCL5 in adjacent peritumoral normal tissues (magnification, x200). H-SCORE, histochemistry score.

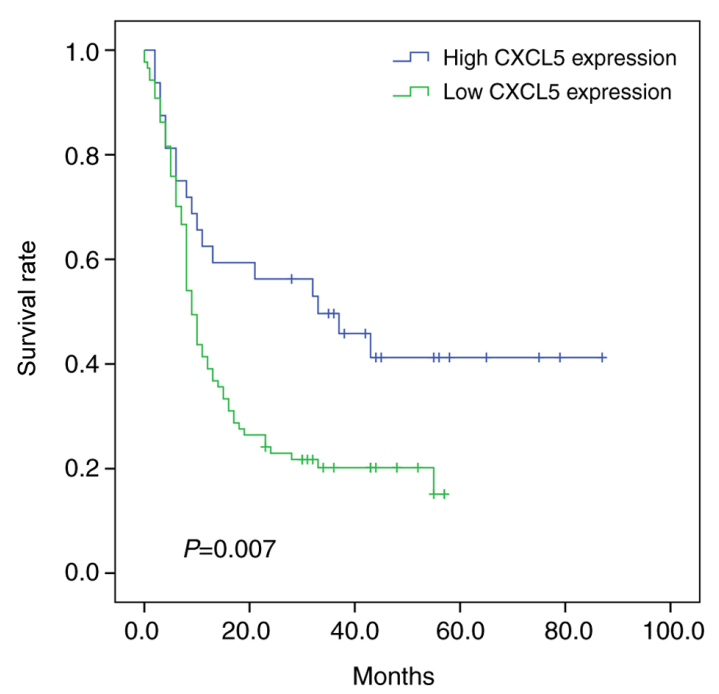

Figure 4. Kaplan-Meier survival curve according to CXCL5 expression levels in cancer tissues of patients with pancreatic cancer.

Association between CXCL5 expression and clinicopathological factors. There was no significant association between the expression of CXCL5 in pancreatic cancer tumor tissues and sex, age, tumor site, tumor site, vascular invasion, 
Table II. Associations between CXCL5 and clinicopathological factors.

Expression level of CXCL5

\begin{tabular}{|c|c|c|c|c|}
\hline & & & & \\
\hline Factors & No. of patients & Low $(\%)$ & High (\%) & P-value \\
\hline Sex & & & & 0.969 \\
\hline Male & 71 & $19(27)$ & $52(73)$ & \\
\hline Female & 48 & $13(27)$ & $35(73)$ & \\
\hline Age, years & & & & 0.589 \\
\hline$<70$ & 81 & $23(28)$ & $58(72)$ & \\
\hline$\geq 70$ & 38 & $9(24)$ & $29(76)$ & \\
\hline Tumor site & & & & 0.879 \\
\hline Head & 72 & $19(26)$ & $53(74)$ & \\
\hline Other & 47 & $13(26)$ & $34(74)$ & \\
\hline VI & & & & 0.116 \\
\hline Yes & 64 & $21(33)$ & $43(67)$ & \\
\hline No & 55 & $11(18)$ & $44(82)$ & \\
\hline Grade & & & & $0.421^{\mathrm{a}}$ \\
\hline 1 & 15 & $6(40)$ & $9(60)$ & \\
\hline 2 & 59 & $16(27)$ & $43(73)$ & \\
\hline 3 & 45 & $10(22)$ & $35(78)$ & \\
\hline $\mathrm{T}$ & & & & 0.139 \\
\hline $\mathrm{T} 1+\mathrm{T} 2$ & 76 & $17(22)$ & $59(78)$ & \\
\hline $\mathrm{T} 3+\mathrm{T} 4$ & 43 & $15(35)$ & $28(65)$ & \\
\hline $\mathrm{N}$ & & & & 0.981 \\
\hline N0 & 63 & 17 (27) & $46(73)$ & \\
\hline $\mathrm{N} 1+\mathrm{N} 2$ & 56 & $15(27)$ & $41(73)$ & \\
\hline $\mathrm{M}$ & & & & 0.854 \\
\hline M0 & 87 & $23(26)$ & $64(74)$ & \\
\hline M1 & 32 & $9(28)$ & $23(72)$ & \\
\hline TNM stage & & & & 0.800 \\
\hline $\mathrm{I}+\mathrm{II}$ & 102 & $27(26)$ & $75(74)$ & \\
\hline III+IV & 17 & $5(29)$ & $12(71)$ & \\
\hline
\end{tabular}

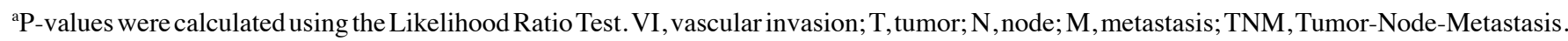

histological grade, $\mathrm{T}$ stage, $\mathrm{N}$ stage, or $\mathrm{M}$ stage $(\mathrm{P}>0.05$; Table II).

Patients with pancreatic cancer with high CXCL5 expression had a poor prognosis: Analysis based on the TMA dataset. OS was used as the prognostic indicator, and Kaplan-Meier survival analysis showed that the prognosis of patients in the CXCL5 high-expression group was significantly worse than that of those in the low-expression group ( $\mathrm{P}=0.007$; Fig. 4). The median survival time of patients in the high-expression group was 9 months with a 95\% CI of 7.6-10.4 months, while the median survival time of patients in the low-expression group was 33 months with a $95 \%$ CI of 7.4-58.6 months.

CXCL5 is an unfavorable independent prognostic factor in patients with pancreatic cancer. Kaplan-Meier survival analysis showed that CXCL5 was significantly associated with the OS of patients with pancreatic cancer. It predicted a poor prognosis in patients with pancreatic cancer $(\mathrm{P}=0.007)$. Among the clinicopathological factors, age $(\mathrm{P}=0.031)$, histological grade $(\mathrm{P}=0.007), \mathrm{N}$ stage $(\mathrm{P}=0.004)$ and Tumor-Node-Metastasis (TNM) stage $(\mathrm{P}=0.002)$ were significantly associated with poor overall survival $(\mathrm{P}<0.05)$. Since the $\mathrm{N}$ stage and the $\mathrm{M}$ stage are factors related to TNM stage, only TNM stage was included in the multifactor regression analysis. Cox multivariate regression analysis showed that age $(\mathrm{P}=0.003)$, histological grade $(\mathrm{P}=0.006)$, TNM stage $(\mathrm{P}=0.002)$ and CXCL5 $(\mathrm{P}=0.011)$ were independent prognostic factors for patients with pancreatic cancer (Table III). Therefore, CXCL5 is an independent factor for a poor prognosis in patients with pancreatic cancer. The hazards ratio (95\% CI) of CXCL5 was 2.003 (1.176-3.412), indicating that in patients with pancreatic cancer, the mortality risk of those with a high expression of CXCL5 in tumor tissues is 2 -fold higher compared with that of those with a low expression. 
Table III. Univariate and multivariate analyses of CXCL5 as a prognostic factor.

\begin{tabular}{|c|c|c|c|c|c|}
\hline \multirow[b]{2}{*}{ Factors } & \multicolumn{2}{|c|}{ Univariate analysis } & \multicolumn{3}{|c|}{ Cox analysis } \\
\hline & MST & P-value & HR & $95 \% \mathrm{CI}$ & P-value \\
\hline Sex (Male/female) & $10 / 10$ & 0.785 & & & \\
\hline Age, years $(<70 / \geq 70)$ & $14 / 8$ & 0.031 & 1.993 & $1.260-3.152$ & 0.003 \\
\hline Grade (I/II/III) & $13 / 8$ & 0.007 & 1.844 & $1.191-2.856$ & 0.006 \\
\hline Tumor site (Head/other) & $9 / 12$ & 0.218 & & & \\
\hline Vascular invasion (No/yes) & $10 / 10$ & 0.729 & & & \\
\hline $\mathrm{T}(\mathrm{T} 1+\mathrm{T} 2 / \mathrm{T} 3+\mathrm{T} 4)$ & $10 / 11$ & 0.220 & & & \\
\hline $\mathrm{N}(\mathrm{N} 0 / \mathrm{N} 1 / \mathrm{N} 2)$ & $15 / 10 / 8$ & 0.004 & & & \\
\hline M (M0/M1) & $12 / 8$ & 0.055 & & & \\
\hline TNM stage $(\mathrm{I}+\mathrm{II} / \mathrm{III}+\mathrm{IV})$ & $13 / 8$ & 0.002 & 2.401 & $1.372-4.200$ & 0.002 \\
\hline CXCL5 (Low/high) & $33 / 9$ & 0.007 & 2.003 & $1.176-3.412$ & 0.011 \\
\hline
\end{tabular}

MST, median survival time; HR, hazards ratio; CI, confidence interval; T, tumor; N, node; M, metastasis; TNM, Tumor-Node-Metastasis.

\section{Discussion}

There are a number of dysregulated chemokines and chemokine receptors in tumor tissues, which serve a role in a variety of cells and are the key regulatory molecules for tumorigenesis, metastasis and immune escape (17). Chemokines and their receptors interact in a variety of non-specific ways, which constitute the multiple ligand and receptor axes (31). They may cause the cell to move directionally and have a regulatory function in a variety of cellular pathology processes and physiological processes (32). With regards to the role of chemokines in the immune system, chemokines are usually divided into inflammatory chemokines and endoenvironmental stability chemokines (33). The latter type of chemokine maintains the stability of the internal environment and is often expressed in the lymphoid homing site. Inflammatory chemokines may recruit immune effector cells to induce an inflammatory microenvironment and are often expressed under pathological conditions in which homeostasis is disrupted (33).

A recent study reported that certain chemokines serve an important role in tumorigenesis; they serve an important role in the migration, invasion, proliferation, chemotherapy resistance, vascular and lymphangiogenesis, distant metastasis and growth of stromal cells in pancreatic cancer (34). Previous studies have reported that the axes of certain chemokines and their receptors not only participate in the regulation of signaling pathways but also regulate the differentiation and infiltration of $\mathrm{T}$ cells, serving a key molecular role in tumor immune escape $(19,20)$. The CXCL12-CXCR4 axis has been identified to have immunosuppressive effects in tumors. Studies have demonstrated that the CXCL12-CXCR4 action axis is associated with a poor prognosis in patients with lung (35), esophageal (36), gastric (37), pancreatic (38), ovarian cancer (39) and other cancer types (40).

Feig et al (41) reported that CXCL12, a chemokine secreted by cancer-associated fibroblasts (CAFs) in pancreatic cancer, may spread and coat the surface of tumor cells, and then
CXCL12 on the surface of tumor cells and mesenchymal CAFs binds to CXCR4, a chemokine receptor on the surface of $\mathrm{T}$ lymphocytes, leading to the clearance of $\mathrm{T}$ lymphocytes from tumor tissues. Karin and Wildbaum (20) have proposed that the CXCL12-CXCR4 axis inhibits the cellular immune response and may induce Th0 cells to differentiate into regulatory $\mathrm{T}$ cells $(\operatorname{Tr} 1)$. At present, there are a variety of inhibitors that may target the CXCL12-CXCR4 axis, including AMD3100 (42), TN14003 (43), and CTCE-9908 (44); therefore, cancer immunotherapy based on cytokines is promising.

CXCL5 is also termed epithelial cell-derived neutrophil-activating protein-78 (45). Studies have reported that CXCL5 has strong effects on granulocyte chemotaxis and angiogenesis $(46,47)$. The target cells of CXCL5 include neutrophils and CXCR $2^{+}$monocytes of CXCR $2^{+}$myeloid-derived suppressor cells (MDSCs) and tumor-associated receptors (48). Therefore, the role of the CXCL5-CXCR2 axis in tumors is also diverse. In colorectal cancer, the preoperative serum CXCL5 level may be used as a novel prognostic marker and is associated with the liver metastasis of colorectal cancer (49). In bone metastatic tumors of prostate cancer, CXCL5 mediates inflammatory cell infiltration and accelerates the growth of metastatic tumors (50). Gao et al (51) reported that the CXCL5-CXCR2 action axis leads to bladder cancer invasion and metastasis by activating the PI3K/AKT signaling pathway to upregulate MMP2/MMP9, suggesting that CXCL5 may be a potential therapeutic target for bladder cancer (51). In thyroid studies, CXCL5 was found to promote the migration and invasion of thyroid cancer cells, affecting tumor epithelial-mesenchymal transition, but not proliferation, suggesting that CXCL5 may affect the microenvironment of thyroid cancer (1). However, in another study, Cui et al (52) suggested that the CXCL5-CXCR2 axis promotes the proliferation of thyroid cancer cells through the P38 and JNK signaling pathways and promotes the progression of the cell cycle from the G1 phase to the S phase (52). Soler-Cardona et al (53) found that the lymph node metastasis rate was significantly increased in melanoma with high 
expression of CXCL5 compared with melanoma with low expression of CXCL5; this may be associated with the fact that CXCL5 recruits PD-1-expressing neutrophils into the tumor microenvironment and interferes with the activation of antitumor immunity $(53,54)$. In other studies, CXCL5 was demonstrated to recruit MDSCs expressing CXCR2 to promote the progression of colon cancer (48) and to promote tumor angiogenesis by increasing the expression of FOXD1 (55). A previous study suggested that high expression of CXCL5 in colorectal cancer is not sufficient to prove it as a diagnostic or prognostic tumor marker (56), but it is considered a potential biomarker and therapeutic target in lung cancer (57,58). A meta-analysis involving 15 studies showed that CXCL5 was an adverse prognostic biomarker in various tumors (59). CXC5 has also been shown to inhibit tumor progression in colon cancer and kidney cancer, which contradicts the aforementioned conclusions (60). At present, there are few studies on CXCL5 in the field of pancreatic cancer. By analyzing three pancreatic cancer data sets from the GEO database, Gu et al (61) obtained 25 core genes, including CXCL5, the functional enrichment of which was demonstrated to be associated with the cell cycle of pancreatic cancer cells, but no further study was conducted on CXCL5 (61).

The present study investigated the significance of CXCL5 in pancreatic cancer. Initially, potential chemokines associated with the prognosis of patients with pancreatic cancer were searched for in the GEO and TCGA databases. CXCL5 was found to be highly expressed in pancreatic cancer tissues and was associated with a poor prognosis. Furthermore, the expression of CXCL5 in the tumor tissues and adjacent peritumoral normal tissues of 119 patients with pancreatic cancer was analyzed by immunohistochemistry. The present study reported that the expression of CXCL5 in pancreatic cancer tumor tissues is higher than that in adjacent peri-tumoral normal tissues, suggesting that CXCL5 is involved in certain pancreatic cancer biological processes. Finally, Kaplan-Meier survival analysis and Cox multivariate analysis revealed that CXCL5 is an independent prognostic factor in patients with pancreatic cancer. High expression of CXCL5 in tumor tissues predicts a poor prognosis and may be a potential biomarker and therapeutic target for pancreatic cancer.

The chemokine, CXCL5, is highly expressed in pancreatic cancer tissues, and high CXCL5 expression is associated with a poor prognosis in patients with pancreatic cancer. The association between CXCL5 and tumor-infiltrating lymphocytes requires investigation in the future.

\section{Acknowledgements}

Not applicable.

\section{Funding}

The present study was supported by the Social Development Research and Demonstration Application Project of the Bureau of Science and Technology of Jiaxing City (grant no. 2019AY32025) and the Medical and Health Science and Technology Project from the Health Committee of Provincial Zhejiang (grant no. 2020K Y951).

\section{Availability of data and materials}

The datasets used and/or analyzed during the current study are available in The Cancer Genome Atlas (cancergenome.nih. gov), the Gene Expression Ominibus database (ncbi.nlm.nih. gov/gds) and GEPIA (Gene Expression Profiling Interactive Analysis, http://gepia.cancer-pku.cn). Tissue microarray and the corresponding data were obtained from ShanghaiOutdo Biotech, China and Shanghai National Engineering Research Centre for Biochips.

\section{Authors' contributions}

BW, ZZ and YS designed the present study. BW and MZ performed the bioinformatics analysis. FC performed the immunohistochemical staining. XW and JW performed the analysis of the immunohistochemical staining and wrote the manuscript. All authors read and approved the final manuscript.

\section{Ethics approval and consent to participate}

Not applicable.

\section{Patient consent for publication}

Not applicable.

\section{Competing interests}

The authors declare that they have no competing interests.

\section{References}

1. 1. Siegel RL, Miller KD and Jemal A: Cancer statistics, 2020. CA Cancer J Clin 70: 7-30, 2020.

2. Saif MW: Pancreatic neoplasm in 2012: An update. Tissue is an issue. JOP 13: 124-127, 2012.

3. Ansari D, Tingstedt B, Andersson B, Holmquist F, Sturesson C, Williamsson C, Sasor A, Borg D, Bauden M and Andersson R: Pancreatic cancer: Yesterday, today and tomorrow. Future Oncol 12: 1929-1946, 2016.

4. Lin QJ, Yang F, Jin C and Fu DL: Current status and progress of pancreatic cancer in China. World J Gastroenterol 21: 7988-8003, 2015.

5. Aroldi F, Bertocchi P, Rosso E, Prochilo T and Zaniboni A: Pancreatic cancer: Promises and failures of target therapies. Rev Recent Clin Trials 11: 33-38, 2016.

6. DeSantis CE, Ma J, Gaudet MM, Newman LA, Miller KD, Goding Sauer A, Jemal A and Siegel RL: Breast cancer statistics, 2019. CA Cancer J Clin 69: 438-451, 2019.

7. Wu T and Dai Y: Tumor microenvironment and therapeutic response. Cancer Lett 387: 61-68, 2017.

8. Frankel T, Lanfranca MP and Zou W: The role of tumor microenvironment in cancer immunotherapy. Adv Exp Med Biol 1036: 51-64, 2017.

9. Ino Y, Yamazaki-Itoh R, Shimada K, Iwasaki M, Kosuge T, Kanai Y and Hiraoka N: Immune cell infiltration as an indicator of the immune microenvironment of pancreatic cancer. Br J Cancer 108: 914-923, 2013.

10. Hinshaw DC and Shevde LA: The tumor microenvironment innately modulates cancer progression. Cancer Res 79: 4557-4566, 2019

11. Wu J, Chen J, Feng Y, Tian H and Chen X: Tumor microenvironment as the 'regulator' and 'target' for gene therapy. J Gene Med 21: e3088, 2019.

12. Ho WJ, Jaffee EM and Zheng L: The tumour microenvironment in pancreatic cancer-clinical challenges and opportunities. Nat Rev Clin Oncol 17: 527-540, 2020. 
13. Dougan SK: The pancreatic cancer microenvironment. Cancer J 23: 321-325, 2017.

14. Tang Y, Xu X, Guo S, Zhang C, Tang Y, Tian Y, Ni B, Lu B and Wang $\mathrm{H}$ : An increased abundance of tumor-infiltrating regulatory $\mathrm{T}$ cells is correlated with the progression and prognosis of pancreatic ductal adenocarcinoma. PLoS One 9: e91551, 2014.

15. Liu L, Zhao G, Wu W, Rong Y, Jin D, Wang D, Lou W and Qin X: Low intratumoral regulatory $\mathrm{T}$ cells and high peritumoral CD8(+) $\mathrm{T}$ cells relate to long-term survival in patients with pancreatic ductal adenocarcinoma after pancreatectomy. Cancer Immunol Immunother 65: 73-82, 2016.

16. Lacalle RA, Blanco R, Carmona-Rodríguez L, Martín-Leal A, Mira E and Mañes S: Chemokine receptor signaling and the hallmarks of cancer. Int Rev Cell Mol Biol 331: 181-244, 2017.

17. Nagarsheth N, Wicha MS and Zou W: Chemokines in the cancer microenvironment and their relevance in cancer immunotherapy. Nat Rev Immunol 17: 559-572, 2017.

18. Bian X, Xiao YT, Wu T, Yao M, Du L, Ren S and Wang J: Microvesicles and chemokines in tumor microenvironment: Mediators of intercellular communications in tumor progression. Mol Cancer 18: 50, 2019.

19. Tan KW, Evrard M, Tham M, Hong M, Huang C, Kato M, Prevost-Blondel A, Donnadieu E, Ng LG and Abastado JP: Tumor stroma and chemokines control T-cell migration into melanoma following Temozolomide treatment. Oncoimmunology 4 e978709, 2015.

20. Karin N and Wildbaum G: The role of chemokines in shaping the balance between CD4(+) T Cell subsets and its therapeutic implications in autoimmune and cancer diseases. Front Immunol 6: 609, 2015

21. Meng W, Xue S and Chen Y: The role of CXCL12 in tumor microenvironment. Gene 641: 105-110, 2018.

22. Zhang J, Wang YF, Wu B, Zhong ZX, Wang KX, Yang LQ, Wang YQ, Li YQ, Gao J and Li ZS: Intraepithelial attack rather than intratumorally infiltration of CD8+T lymphocytes is a favorable prognostic indicator in pancreatic ductal adenocarcinoma. Curr Mol Med 17: 689-698, 2017.

23. Haider S, Wang J, Nagano A, Desai A, Arumugam P, Dumartin L, Fitzgibbon J, Hagemann T, Marshall JF, Kocher HM, et al: A multi-gene signature predicts outcome in patients with pancreatic ductal adenocarcinoma. Genome Med 6: 105, 2014.

24. Wang J, Dumartin L, Mafficini A, Ulug P, Sangaralingam A Alamiry NA, Radon TP, Salvia R, Lawlor RT, Lemoine NR, et al: Splice variants as novel targets in pancreatic ductal adenocarcinoma. Sci Rep 7: 2980, 2017.

25. Zhang G, He P, Tan H, Budhu A, Gaedcke J, Ghadimi BM, Ried T, Yfantis HG, Lee DH, Maitra A, et al: Integration of metabolomics and transcriptomics revealed a fatty acid network exerting growth inhibitory effects in human pancreatic cancer. Clin Cancer Res 19: 4983-4993, 2013

26. Zhang G, Schetter A, He P, Funamizu N, Gaedcke J, Ghadimi BM, Ried T, Hassan R, Yfantis HG, Lee DH, et al: DPEP1 inhibits tumor cell invasiveness, enhances chemosensitivity and predicts clinical outcome in pancreatic ductal adenocarcinoma. PLoS One 7: e31507, 2012.

27. Tang J, Wang Y, Luo Y, Fu J, Zhang Y, Li Y, Xiao Z, Lou Y, Qiu Y and Zhu F: Computational advances of tumor marker selection and sample classification in cancer proteomics. Comput Struct Biotechnol J 18: 2012-2025, 2020.

28. $\mathrm{R}$ Core Team: R: A language and environment for statistical computing. $R$ Foundation for Statistical Computing, Vienna, Austria, 2014. URL http://www.R-project.org/.

29. Chen H and Boutros PC: VennDiagram: A package for the generation of highly-customizable Venn and Euler diagrams in R. BMC Bioinformatics 12: 35, 2011.

30. Allen PJ, Kuk D, Castillo CF, Basturk O, Wolfgang CL, Cameron JL, Lillemoe KD, Ferrone CR, Morales-Oyarvide V, He J, et al: Multi-institutional validation study of the American joint commission on cancer (8th edition) changes for $\mathrm{T}$ and $\mathrm{N}$ staging in patients with pancreatic adenocarcinoma. Ann Surg 265: 185-191, 2017.

31. Legler DF and Thelen M: Chemokines: Chemistry, biochemistry and biological function. Chimia (Aarau) 70: 856-859, 2016.

32. Luster AD: Chemokines-chemotactic cytokines that mediate inflammation. N Engl J Med 338: 436-445, 1998.

33. Zlotnik A and Yoshie O: The chemokine superfamily revisited Immunity 36: 705-716, 2012.

34. Wu PF, Lu ZP, Cai BB, Tian L, Zou C, Jiang KR and Miao Y: Role of CXCL12/CXCR4 signaling axis in pancreatic cancer. Chin Med J (Engl) 126: 3371-3374, 2013.
35. Wald O, Shapira OM and Izhar U: CXCR4/CXCL12 axis in non small cell lung cancer (NSCLC) pathologic roles and therapeutic potential. Theranostics 3: 26-33, 2013.

36. Goto M and Liu M: Chemokines and their receptors as biomarkers in esophageal cancer. Esophagus 17: 113-121, 2020.

37. Lee HJ and Jo DY: The role of the CXCR4/CXCL12 axis and its clinical implications in gastric cancer. Histol Histopathol 27: 1155-1161, 2012.

38. Sleightholm RL, Neilsen BK, Li J, Steele MM, Singh RK, Hollingsworth MA and Oupicky D: Emerging roles of the CXCL12/CXCR4 axis in pancreatic cancer progression and therapy. Pharmacol Ther 179: 158-170, 2017.

39. Salomonnson E, Stacer AC, Ehrlich A, Luker KE and Luker GD: Imaging CXCL12-CXCR4 signaling in ovarian cancer therapy. PLoS One 8: e51500, 2013.

40. Samarendra H, Jones K, Petrinic T, Silva MA, Reddy S, Soonawalla $Z$ and Gordon-Weeks A: A meta-analysis of CXCL12 expression for cancer prognosis. Br J Cancer 117: 124-135, 2017.

41. Feig C, Jones JO, Kraman M, Wells RJ, Deonarine A, Chan DS, Connell CM, Roberts EW, Zhao Q, Caballero OL, et al: Targeting CXCL12 from FAP-expressing carcinoma-associated fibroblasts synergizes with anti-PD-L1 immunotherapy in pancreatic cancer. Proc Natl Acad Sci USA 110: 20212-20217, 2013.

42. Rizvi NA, Hellmann MD, Snyder A, Kvistborg P, Makarov V, Havel JJ, Lee W, Yuan J, Wong P, Ho TS, et al: Cancer immunology. Mutational landscape determines sensitivity to PD-1 blockade in non-small cell lung cancer. Science 348: 124-128, 2015.

43. Beider K, Begin M, Abraham M, Wald H, Weiss ID, Wald O, Pikarsky E, Zeira E, Eizenberg O, Galun E, et al: CXCR4 antagonist 4F-benzoyl-TN14003 inhibits leukemia and multiple myeloma tumor growth. Exp Hematol 39: 282-292, 2011.

44. Wong D, Kandagatla P, Korz W and Chinni SR: Targeting CXCR4 with CTCE-9908 inhibits prostate tumor metastasis. BMC Urol 14: 12, 2014.

45. Okabe H, Beppu T, Ueda M, Hayashi H, Ishiko T, Masuda T, Otao R, Horlad H, Mima K, Miyake K, et al: Identification of CXCL5/ENA-78 as a factor involved in the interaction between cholangiocarcinoma cells and cancer-associated fibroblasts. Int J Cancer 131: 2234-2241, 2012.

46. Walz A, Burgener R, Car B, Baggiolini M, Kunkel SL and Strieter RM: Structure and neutrophil-activating properties of a novel inflammatory peptide (ENA-78) with homology to interleukin 8. J Exp Med 174: 1355-1362, 1991.

47. Keane MP, Belperio JA, Xue YY, Burdick MD and Strieter RM: Depletion of CXCR2 inhibits tumor growth and angiogenesis in a murine model of lung cancer. J Immunol 172: 2853-2860, 2004.

48. Katoh H, Wang D, Daikoku T, Sun H, Dey SK and Dubois RN: CXCR2-expressing myeloid-derived suppressor cells are essential to promote colitis-associated tumorigenesis. Cancer Cell 24: 631-644, 2013.

49. Kawamura M, Toiyama Y, Tanaka K, Saigusa S, Okugawa Y, Hiro J, Uchida K, Mohri Y, Inoue Y and Kusunoki M: CXCL5, a promoter of cell proliferation, migration and invasion, is a novel serum prognostic marker in patients with colorectal cancer. Eur J Cancer 48 : 2244-2251, 2012.

50. Roca H, Jones JD, Purica MC, Weidner S, Koh AJ, Kuo R, Wilkinson JE, Wang Y, Daignault-Newton S, Pienta KJ, et al: Apoptosis-induced CXCL5 accelerates inflammation and growth of prostate tumor metastases in bone. J Clin Invest 128: 248-266, 2018.

51. Gao Y, Guan Z, Chen J, Xie H, Yang Z, Fan J, Wang X and Li L: CXCL5/CXCR2 axis promotes bladder cancer cell migration and invasion by activating PI3K/AKT-induced upregulation of MMP2/MMP9. Int J Oncol 47: 690-700, 2015.

52. Cui D, Zhao Y and Xu J: Activation of CXCL5-CXCR2 axis promotes proliferation and accelerates $\mathrm{G} 1$ to $\mathrm{S}$ phase transition of papillary thyroid carcinoma cells and activates JNK and p38 pathways. Cancer Biol Ther 20: 608-616, 2019.

53. Soler-Cardona A, Forsthuber A, Lipp K, Ebersberger S, Heinz M, SchossleitnerK, Buchberger E, Gröger M,Petzelbauer P, Hoeller C, et al: CXCL5 facilitates melanoma cell-neutrophil interaction and lymph node metastasis. J Invest Dermatol 138: 1627-1635, 2018.

54. Forsthuber A, Lipp K, Andersen L, Ebersberger S, Graña-Castro O, Ellmeier W, Petzelbauer P, Lichtenberger BM and Loewe R: CXCL5 as regulator of neutrophil function in cutaneous melanoma. J Invest Dermatol 139: 186-194, 2019.

55. Chen C, Xu ZQ, Zong YP, Ou BC, Shen XH, Feng H, Zheng MH, Zhao JK and Lu AG: CXCL5 induces tumor angiogenesis via enhancing the expression of FOXD1 mediated by the AKT/NF- $\mathrm{BB}$ pathway in colorectal cancer. Cell Death Dis 10: 178, 2019. 
56. Yildirim K, Colak E, Aktimur R, Gun S, Taskin MH Nigdelioglu A, Aktimur SH, Karagöz F and Ozlem N: Clinical value of CXCL5 for determining of colorectal cancer. Asian Pac J Cancer Prev 19: 2481-2484, 2018.

57. Wang L, Shi L, Gu J, Zhan C, Xi J, Ding J and Ge D: CXCL5 regulation of proliferation and migration in human non-small cell lung cancer cells. J Physiol Biochem 74: 313-324, 2018.

58. Wu K, Yu S, Liu Q, Bai X, Zheng X and Wu K: The clinical significance of CXCL5 in non-small cell lung cancer. Onco Targets Ther 10: 5561-5573, 2017.

59. Hu B, Fan H, Lv X, Chen S and Shao Z: Prognostic significance of CXCL5 expression in cancer patients: A meta-analysis. Cancer Cell Int 18: 68, 2018.
60. Speetjens FM, Kuppen PJ, Sandel MH, Menon AG, Burg D, van de Velde CJ, Tollenaar RA, de Bont HJ and Nagelkerke JF: Disrupted expression of CXCL5 in colorectal cancer is associated with rapid tumor formation in rats and poor prognosis in patients. Clin Cancer Res 14: 2276-2284, 2008.

61. Gu Y, Feng Q, Liu H, Zhou Q, Hu A, Yamaguchi T, Xia S and Kobayashi H: Bioinformatic evidences and analysis of putative biomarkers in pancreatic ductal adenocarcinoma. Heliyon 5: e02378, 2019.

(i) $($ This work is licensed under a Creative Common Attribution-NonCommercial-NoDerivatives 4.0 International (CC BY-NC-ND 4.0) License. 